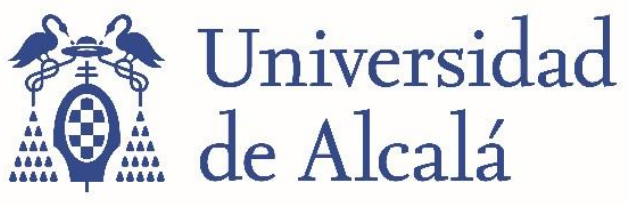

BIBLIOTECA

Document downloaded from the institutional repository of the University of Alcala: http://ebuah.uah.es/dspace/

This is a postprint version of the following published document:

Shen, Li-Yong, Pérez-Díaz, S., Goldman, R. \& Feng, Y. 2019, "Representing rational curve segments and surface patches using semi-algebraic sets", Computer Aided Geometric Design, vol. 74, article 101770

Available at https://doi.org/10.1016/j.cagd.2019.101770

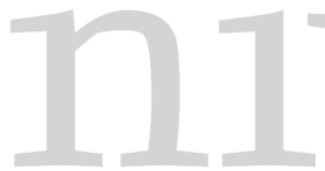

(C) 2019 Elsevier

(Article begins on next page)

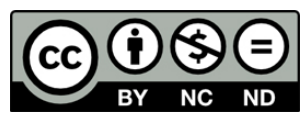

This work is licensed under a

Creative Commons Attribution-NonCommercial-NoDerivatives

4.0 International License. 
7 Abstract

We provide a framework for representing segments of rational planar curves or patches of rational tensor product surfaces with no singularities using semi-algebraic sets. Given a rational planar curve segment or a rational tensor product surface patch with no singularities, we find the implicit equation of the corresponding unbounded curve or surface and then construct an algebraic box defined by some additional equations and inequalities associated to the implicit equation. This algebraic box is proved to include only the given curve segment or surface patch without any extraneous parts of the unbounded curve or surface. We also explain why it is difficult to construct such an algebraic box if the curve segment or surface patch includes some singular points such as self-intersections. In this case, we show how to isolate a neighborhood of these special points from the corresponding curve segment or surface patch and to represent these special points with small curve segments or surface patches. This framework allows us to dispense with expensive approximation methods such a voxels for representing surface patches.

\section{Representing rational curve segments and surface patches using semi-algebraic sets}

\author{
Li-Yong Shen ${ }^{\mathrm{a}, *}$, Sonia Pérez-Díaz ${ }^{\mathrm{b}}$, Ron Goldman ${ }^{\mathrm{c}}$, Yifei Feng ${ }^{\mathrm{a}}$ \\ ${ }^{a}$ School of Mathematical Sciences, University of Chinese Academy of Sciences, 100049, Beijing, China \\ ${ }^{b}$ Grupo ASYNACS, Dpto. de Física y Matemáticas, Universidad de Alcalá, 28871-Alcalá de Henares, Madrid, Spain \\ ${ }^{c}$ Computer Science Department, Rice University, 6100, Main St., MS-132, TX 77005, USA
}

Keywords: Rational curve segment, Rational surface patch, Semi-algebraic set, Implicitization

\section{Introduction}

Rational curve segments or surface patches are widely used in Computer Aided Geometric Design, Computer Graphics, and Computer Aided Design (CAD). The most popular representations in CAD are Bspline curves/surfaces and each curve segment/surface patch can be reduced to Bézier form. The parametric expression is best for rendering, since using the parametric form it is easy to generate many points on the curve or surface. But to determine whether a point lies on a curve or surface, it is easier to use the implicit expression for the curve or surface. Implicitization means finding an implicit representation starting from a parametric representation. When the parametrization is rational, the implicit equation is a polynomial equation in the Cartesian coordinates.

Because of their many fundamental applications, implicitization algorithms for rational curves and surfaces have been the focus of much attention over the past several decades (Buchberger, 1985; Busé, 2014; Chen et al., 2005; Manocha and Canny, 1992; Elkadi and Mourrain, 2004; Sederberg and Chen, 1995; Shen and Goldman, 2017, 2018; Yao et al., 2019). Among these many possible techniques, the method of Gröbner bases (Buchberger, 1985) is well-known in academic circles, since this method is theoretically complete. However, Gröbner bases have exponential computational complexity and hence are highly inefficient. Instead, researchers have focused their attention on implicitization methods based on matrix constructions and resultant calculations (Busé, 2014; Chen et al., 2005; Manocha and Canny, 1992; Sederberg and Chen,

\footnotetext{
* Corresponding author

Email addresses: lyshen@ucas.ac.cn (Li-Yong Shen), sonia.perez@uah.es (Sonia Pérez-Díaz), rng@rice.edu (Ron Goldman), fengyifei15@mails.ucas.ac.cn (Yifei Feng)
} 
1995; Shen and Goldman, 2017, 2018), which are much more efficient than Gröbner basis computations. General implicitization methods generate algorithms for a given curve or surface. However, we need take care of the boundaries when we focus on a curve segment or a surface patch. It is not a trivial task to find implicitization algorithms for rational curves and surfaces where the parameters are not bounded and then it is even more difficult to find an exact implicit representation for a rational curve segment or a surface patch with bounded parameters.

There are, however, alternative implicit representations for curves and surfaces such as radial basis functions (RBF) (Carr et al., 2001; Buhmann, 2003) and voxelizations method (Cohen-Or and Kaufman, 1995; Laine, 2013). A radial basis function is a real-valued function whose value depends only on the distance from the origin. Sums of radial basis functions are typically used to approximate functions. An indispensable stage in 3-dimensional computer graphics is the synthesis of voxel representations of 3-dimensional objects. This stage is called voxelization and is concerned with converting geometric objects from their smooth shape representation into a set of voxels that best approximates the smooth object. Both of these methods are designed as approximation techniques and can be used for curve segments and surface patches. But these methods may then introduce a large amount of parametric data or mesh segments for some important geometric features.

In this paper, we represent curve segments or surface patches with no singularities by semi-algebraic sets whose equations and inequalities are computed using the latest implicitization algorithms. For a given curve segment or surface patch with no singularities, we find the implicit equation of the corresponding rational curve or surface without boundaries and construct as well an algebraic box defined by some equations and inequalities associated to the implicit equation. The constructed algebraic box is proved to include only the expected curve segment or surface patch without any extraneous parts of the curve or surface. Using our constructions, the parametric expressions of the bounding curve segments or surface patches of the semi-algebraic set can be found directly. Further discussions show that it is difficult to construct a simple bounding algebraic box if the curve segment or surface patch includes some special points such as self intersection points. In this case, we prefer to isolate the neighborhood of this point from the curve segment or surface patch.

The paper is organized as follows. In Section 2, we briefly recall the latest implicitization methods for planar rational curves and rational surfaces. In Section 3, we construct the semi-algebraic set for a parametric planar curve segment with no singularities. In section 4 , we deal with the corresponding problem for surface patches with no singularities. Finally, we conclude our paper in Section 4 with a brief summary of our work along with a brief review of the motivation for this research.

\section{Implicitization}

Representing rational curve segments and surface patches using semi-algebraic sets is based on implicitization for rational curves and surfaces. Hence, here we briefly review algorithms to implicitize rational curves and surfaces.

Consider a rational planar curve in homogeneous form

$$
\mathcal{P}(s)=\left(p_{1}(s), p_{2}(s), p_{3}(s)\right)
$$

where $p_{1}(s), p_{2}(s), p_{3}(s)$ are linearly independent polynomials of degree $n$ with no common factors. Let $F(x, y, w)=0$ be the implicit equation corresponding to the rational parametrization. Then the nonzero homogeneous polynomial $F(x, y, w)$ is irreducible and $F\left(p_{1}(s), p_{2}(s), p_{3}(s)\right) \equiv 0$. To implicitize rational planar curves, we can use either the technique of moving lines (Sederberg and Chen, 1995) or the method of $\mu$-bases (Chen and Wang, 2002).

Consider next a rational tensor product surface of bidegree $(m, n)$ in homogeneous form

$$
\mathcal{P}(s, t)=\left(p_{1}(s, t), p_{2}(s, t), p_{3}(s, t), p_{4}(s, t)\right)
$$


where $p_{1}(s, t), p_{2}(s, t), p_{3}(s, t), p_{4}(s, t)$ are linearly independent polynomials of bidegree $(m, n)$ with no common factors. Let $F(x, y, z, w)=0$ be the implicit equation corresponding to the rational parametrization. Then the nonzero homogeneous polynomial $F(x, y, z, w)$ is irreducible and $F\left(p_{1}(s, t), p_{2}(s, t), p_{3}(s, t)\right.$, $\left.p_{4}(s, t)\right) \equiv 0$.

The latest implicitzation method works correctly and efficiently even in the presence of base points by combining three complementary approaches to implicitization (Shen and Goldman, 2018). The algorithm first tries the Dixon $\mathcal{A}$-resultant and univariate resultant methods (Chionh, 2001; Shen and Yuan, 2010), which work efficiently for some rational parametrizations with no base points as well as for some surfaces with very special configurations of base points. If these methods fail, the algorithm invokes the method of moving planes and moving quadrics for surfaces with simple base points, and some other cases that have sufficiently many moving planes and moving quadrics that follow the surface (Sederberg and Chen, 1995). For the remaining surfaces, we employ the latest method, which takes the resultant of three moving planes generated by three syzygies of low bidegrees and is simpler and more efficient than the $\mu$-basis method (Shen and Goldman, 2017).

We have implemented a Maplesoft package for implicitizing rational planar curves and rational tensor product surfaces, developed using the preceding implicitization techniques (Yao et al., 2019). From our experiments, we find that this package can compute the implicit equation of any bicubic tensor product surface efficiently. The results for implicitizing all the patches of the Utah teapot can be found in (Shen and Goldman, 2017) and additional experiments can be found in (Shen and Goldman, 2018). For ruled surfaces, the package uses a more efficient way to compute the implicit equation based on univariate resultants.

\section{Curve Segments}

A curve segment is often given in parametric form by restricting the parameter to a finite interval

$$
C_{p}=\{(x, y, w) \mid(x, y, w)=\mathcal{P}(s), s \in[0,1]\} .
$$

To find a semi-algebraic set to represent this curve segment, we first find the implicit equation of the rational curve

$$
C=\{(x, y, w) \mid F(x, y, w)=0\} .
$$

The boundary points of $C_{p}$ are $\mathbf{p}_{1}=\mathcal{P}(0), \mathbf{p}_{2}=\mathcal{P}(1)$. To construct a semi-algebraic set to bound this curve segment, we generate two lines $L_{1}$ and $L_{2}$ passing through these two points. For instance, we can set $L_{1}:=\left((x, y, w)-\mathbf{p}_{1}\right) \cdot \mathbf{v}_{0}=0$ and $L_{2}:=\left((x, y, w)-\mathbf{p}_{2}\right) \cdot \mathbf{v}_{0}=0$ where $\mathbf{v}_{0}$ is a vector selected (see next paragraph and Lemma 1$)$ so that the we can finally construct a bounding semi-algebraic set.

To construct a region including the curve segment $C_{p}$, we introduce two additional curves $\mathcal{P}_{i}(s)=$ $\mathcal{P}(s)+(-1)^{i} \varepsilon \mathbf{v}_{0}, s \in[0,1]$, which we denote by $C_{F_{i}, p}$. The implicit equations of $\mathcal{P}_{i}$ can be found directly as $F_{i}(x, y, w)=F\left((x, y, w)+(-1)^{i} \mathbf{v}_{0}\right)=0$ and these polynomials define the curves $C_{i}=\{(x, y, w) \mid$ $\left.F_{i}(x, y, w)=0\right\}$. The construction of the curves $\mathcal{P}_{i}$ is similar to finding an offset, the vector $\mathbf{v}_{0}$ can be selected as the mean of the normal vectors of $C_{p}$. Now we define a semi-algebraic set

$$
C_{a}=\left\{(x, y, w) \mid F(x, y, w)=0, L_{i}(x, y, w) \geq 0, F_{i}(x, y, w)>0, i=1,2\right\}
$$

associated to the curve segment $C_{p}$. Notice that $\mathbf{p}_{i}+\varepsilon \mathbf{v}_{0}-2 u \varepsilon \mathbf{v}_{0}, u \in[0,1]$ are the parametric forms of the segments of $L_{i}$ and these expressions define curve segments $C_{L_{i}, p}=\left\{(x, y, w)=u\left(\mathbf{p}_{i}-\varepsilon \mathbf{v}_{0}\right)+(1-\right.$ $\left.u)\left(\mathbf{p}_{i}+\varepsilon \mathbf{v}_{0}\right)=\mathbf{p}_{i}+\varepsilon \mathbf{v}_{0}-2 u \varepsilon \mathbf{v}_{0}, u \in[0,1]\right\}$. We will soon show (see Propositions 1 and 2) that under certain conditions, the curve segment can be represented as this semi-algebraic set, i.e., $C_{p}=C_{a}$ (see Figure 1).

Note that $C$ is the closure of the curve defined by any parametrization. To ensure that only $C_{p}$, and no other segments of $C$, is included in the semi-algebraic set $C_{a}$, we need to consider some feature points of the curve $C$.

The singular points of the implicit curve satisfy $\frac{\partial F}{\partial x}=\frac{\partial F}{\partial y}=\frac{\partial F}{\partial w}=F(x, y, w)=0$. If a singular point is on the curve segment $C_{p}$, we introduce a neighborhood isolating box for this point and approximate the 


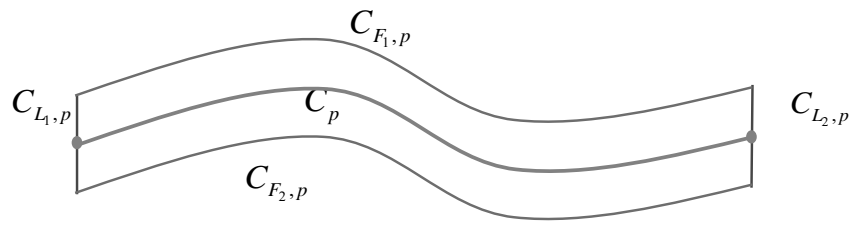

Figure 1: A curve segment included in a semi-algebraic set

curve segment in this box with a simple curve segment. For instance, we can use a cubic segment such that the segment has $C^{2}$ continuity at the two end points with the original curve.

If the singular point does not lie on the curve segment $C_{p}$ but is included in the semi-algebraic set $C_{a}$, we need to remove this point from the semi-algebraic set.

There may be points with real coordinates on $C$ but associated to the complex parameters, that is, there may exist complex parameters $t_{c}=r+c J, J^{2}=-1, c \neq 0$ such that $\mathcal{P}\left(t_{c}\right) \in \mathbb{R}^{3}$. The zero condition of the imaginary parts $\Im\left(\frac{p_{1}\left(t_{c}\right)}{p_{3}\left(t_{c}\right)}\right)=\Im\left(\frac{p_{2}\left(t_{c}\right)}{p_{3}\left(t_{c}\right)}\right)=0$ leads to a polynomial system $f_{1}(r, c)=f_{2}(r, c)=0$, where $\Im(\cdot)$ returns the imaginary part. Then by Bezout's theorem this point set is generally zero dimensional if $f_{1}$ and $f_{2}$ are coprime. This system can be solved using a technique similar to Cylindrical Algebraic Decomposition (Caviness and Johnson, 1998). Briefly, one can eliminate one variable by computing the resultant with respect to this variable, then find the real roots of this resultant, and finally lift the real roots to the solutions of the system. There are also efficient methods that can be found in (Cheng and Jin, 2015; Sagraloff and Mehlhorn, 2016).

For general applications, we always assume that the parameters are real numbers. If this kind of point corresponding to a complex parameter does not lie on the curve segment $C_{p}$, we need to remove this point from the semi-algebraic set.

Proposition 1. Let $C_{p}=\{(x, y, w) \mid(x, y, w)=\mathcal{P}(s), s \in[0,1]\}$ be a segment of a rational curve and let $C_{a}=\left\{(x, y, w) \mid F(x, y, w)=0, L_{i}(x, y, w) \geq 0, F_{i}(x, y, w)>0, i=1,2\right\}$ be given as above. If

1) There are no singular points in $C_{p}$;

2) Each boundary line $L_{i}$ and $C_{p}$ are not tangent at $\mathbf{p}_{i}$;

3) Each boundary line $L_{i}$ and $C_{p}$ have no intersection point other than $\mathbf{p}_{i}$;

4) Each boundary curve $C_{i}$ and $C_{p}$ have no intersection point;

5) Each boundary line segment $C_{L_{i}, p}$ and the curve $C$ have no intersection point other than $\mathbf{p}_{i}$;

6) Each boundary curve segment $C_{F_{i}, p}$ and the curve $C$ have no intersection point,

then the semi-algebraic set $C_{a}$ is equivalent to the curve segment $C_{p}$.

Proof. By conditions 3 ) and 4), all the points of the curve segment $C_{p}$ lie in the same real region determined by the boundary lines or curves of $C_{a}$, so $C_{p} \subset C_{a}$.

We now show that $C_{p} \supset C_{a}$. Suppose that there exists a point $\mathbf{q}_{0} \in C_{a} \backslash C_{p}$ and select another point $\mathbf{q}_{1} \in C_{p}$. Then there exists a path $\widetilde{\mathbf{q}_{0} \mathbf{q}_{1}}$ in $C$ connecting $\mathbf{q}_{0}$ and $\mathbf{q}_{1}$, since $C$ is a rational curve. The path curve $\widetilde{\mathbf{q}_{0} \mathbf{q}_{1}}$ is totally included in $C_{a}$, otherwise $\widetilde{\mathbf{q}_{0} \mathbf{q}_{1}}$ has intersections with at least one boundary curve of $C_{L_{i}, p}$ or $C_{F_{i}, p}$, which cannot happen at any point other than an end point $\mathbf{p}_{i}$ by conditions 5) and 6) (see Figure 2). By condition 2), the path curve $\widetilde{\mathbf{q}_{0} \mathbf{q}_{1}}$ does not pass through the endpoints of $C_{p}$ (see Figure 2 , top), so $\widetilde{\mathbf{q}_{0} \mathbf{q}_{1}}$ must leave $C_{p}$ at a certain point $\mathbf{q}_{s}$. Hence $\mathbf{q}_{s}$ is a self-intersection point of $\mathcal{C}_{p}$ and $\mathbf{q}_{s}$ is a singular point of $C_{p}$, a contradiction to condition 1). Therefore there does not exist such a point $\mathbf{q}_{0}$, and so we conclude that $C_{a}=C_{p}$. 
Figure 2 illustrates the possible cases not satisfying the conditions given in Proposition 1.

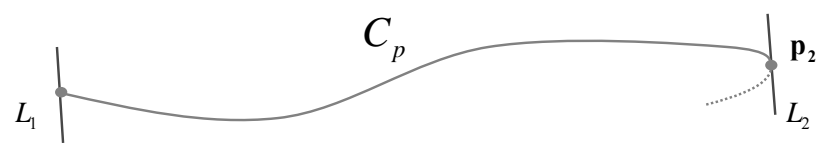

$C_{p}$ tangent to $L_{2}$ at $\mathbf{p}_{2}$

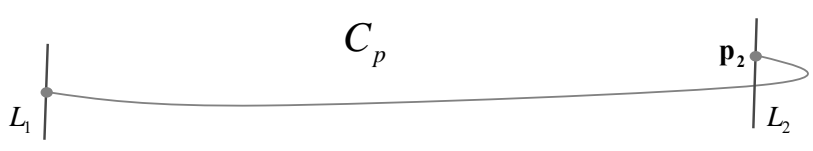

$C_{p}$ and $L_{2}$ have intersections other than $\mathbf{p}_{2}$

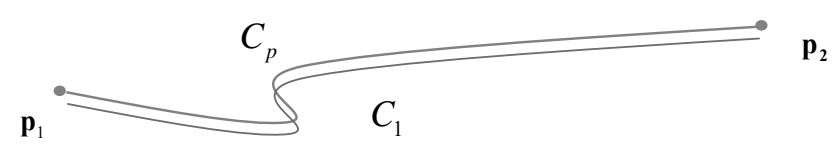

$C_{p}$ and $C_{1}$ intersect

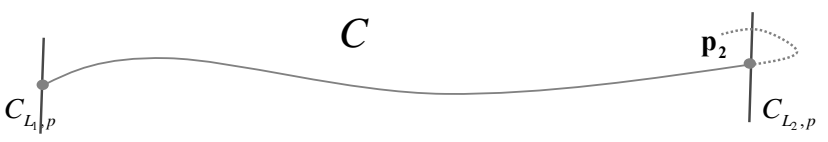

$C$ and $C_{L_{2}, p}$ have intersections other than $\mathbf{p}_{2}$

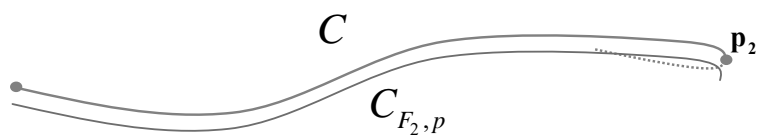

$C$ and $C_{F_{2}, p}$ have intersections other than $\mathbf{p}_{2}$

Figure 2: Excluded cases for the semi-algebraic set for the curve segment

Proposition 2. Let $C_{p}=\{(x, y, w) \mid(x, y, w)=\mathcal{P}(s), s \in[0,1]\}$ be a segment of a rational curve with no singular points. Then this curve segment can be represented by the union of semi-algebraic sets

$$
\bigcup_{k=1}^{m} C_{k, a}=\bigcup_{k=1}^{m}\left\{(x, y, w) \mid F(x, y, w)=0, L_{k i}(x, y, w) \geq 0, F_{k i}(x, y, w)>0, i=1,2 .\right\}
$$

Proof. For a non-singular point $\mathbf{p}_{0}$ associated to the parameter $s_{0}$ and any $\epsilon>0$, consider the curve segment $C_{p, \epsilon}$ with $s \in\left[s_{0}-\epsilon, s_{0}+\epsilon\right]$. One can construct a semi-algebraic set $C_{a, \epsilon}$ for the curve segment $C_{p, \epsilon}$

$$
C_{a, \epsilon}=\left\{(x, y, w) \mid F(x, y, w)=0, L_{i}(x, y, w) \geq 0, F_{i}(x, y, w)>0, i=1,2\right\}
$$


where $\varepsilon_{\epsilon}$ and $\mathbf{v}_{\epsilon}$ are parameters selected for the boundary lines and curves so that they satisfy conditions 2)-6) of Proposition 1. By the Taylor approximation to the curve at $s_{0}$, there exist $\varepsilon_{\epsilon}$ and $\mathbf{v}_{\epsilon}$ for such a semi-algebraic set. By Proposition 1 and the fact that the curve segment is a closed set, we have $C_{a, \epsilon}=C_{p, \epsilon}$. Now by compactness we can find a finite union of semi-algebraic set, say $m$ sets, that cover the entire curve segment.

Remark 1. The number $m$ of the sets is associated to $\epsilon$ and the geometry of curve segment. In fact, the boundary surfaces, determined by the parameters $\varepsilon_{\epsilon}$ and $\mathbf{v}_{\epsilon}$, are also associated to $\epsilon$. So the conditions in Proposition 1 are associated to the curve segment and $\epsilon$.

\subsection{Implicitization Algorithm for Curve Segments}

Lemma 1. For a curve segment $\mathcal{P}(s), s \in[0,1]$ and a vector $\mathbf{v}_{0}$, the curve segments $C_{F_{i}, p}$ and $C_{p}$ have no intersection point if the unit normal vectors of the segment are strictly included in a half circle and $v_{0}$ is a normal vector at any point of $\mathcal{P}(s), s \in[0,1]$.

Proof. Let $v_{0}$ be a normal vector at a point of $\mathcal{P}(s), s \in[0,1]$. A line parallel to $\mathbf{v}_{0}$ has at most one real intersection point with the curve segment $C_{p}$ since the unit normal vectors of the segment are strictly included in half a circle, i.e., the curve segment is a single-valued function relative to the vector $\mathbf{v}_{0}$. Hence, any translational curve segment $C_{F_{i}, p}$ along the $\mathbf{v}_{0}$ has no intersection with $C_{p}$.

Algorithm 1. Find an exact semi-algebraic set representing a rational planar curve segment $\mathcal{P}(s), s \in[0,1]$ having no singular points.

1. Find a vector $\mathbf{v}_{0}$ satisfying Lemma 1 and not parallel to tangent vectors of $\mathbf{p}_{1}, \mathbf{p}_{2}$. If no such $\mathbf{v}_{0}$ exists, subdivide the curve segment until there exist such $\mathbf{v}_{0}$ satisfying Lemma 1 for each subdivided segment.

2. Construct an initial semi-algebraic set for the curve segments by the above set of the form $C_{a}$.

3. Check conditions 3)-6) of Proposition 1 and update $\varepsilon=\varepsilon / 2$ if some conditions do not hold.

Algorithm 2. Find a semi-algebraic set for a rational planar curve segment $\mathcal{P}(s), s \in[0,1]$ with given error.

1. Compute the implicit equation $F(x, y, w)$ of $\mathcal{P}(s)$;

2. Compute the singular points of the curve (Pérez-Díaz, 2007; Jia and Goldman, 2009).

3. For each singular point, construct an isolating box. In the box we approximate the curve segment using a line segment with a given error (Cheng et al., 2009).

4. For a curve segment with no singular points, find an exact semi-algebraic set using Algorithm 1.

Remark 2. The approximation step in Algorithm 2 is needed in order to isolate the singular point and its neighboring region by a box. The approximating line segment can be replaced by a rational curve segment to meet the curve with high continuity. The approximated curve should have no other singular points in the isolating box and then the isolating box can be regarded as the semi-algebraic set for the approximated curve.

With the same error control from the curve to the boundaries of the boxes, the implemented examples show that many fewer semi-algebraic sets are needed relative to the typical mesh method (see Figure 3).

\section{Surface Patches}

There are bounded surfaces such as spheres, tori, cyclides, Steiner surfaces, and surfaces of revolution generated by closed curves. In freeform geometric design, more surfaces are unbounded. Hence we have to study surface patches (see Figure 4, the Utah teapot includes 32 bicubic Bézier surface patches). Generally a surface patch is given in parametric form by restricting the parameters to finite intervals

$$
S_{p}=\{(x, y, z, w) \mid(x, y, z, w)=\mathcal{P}(s, t), s \in[0,1], t \in[0,1]\} .
$$



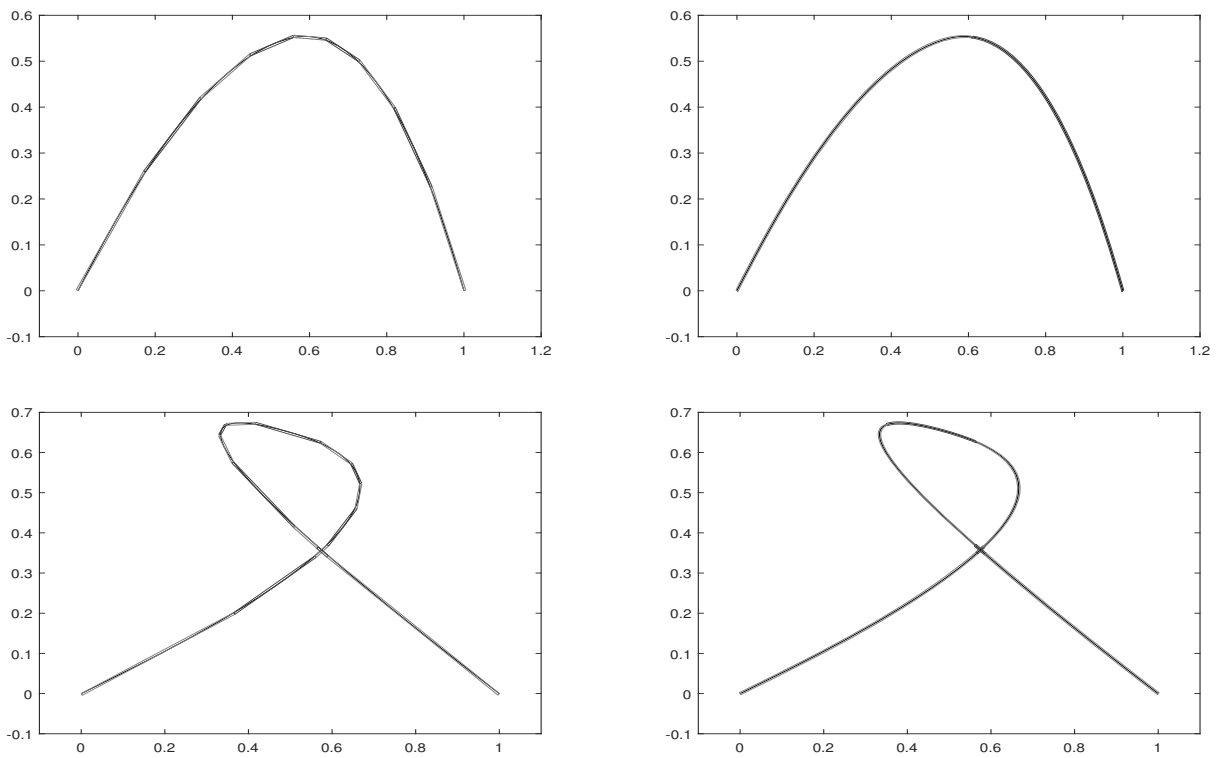

Figure 3: Curve segments bounded by meshes v.s. semi-algebraic boxes

In analogy with rational curves, we would like to find a semi-algebraic set to represent this tensor product surface patch. We first find the implicit equation of the rational surface

$$
S=\{(x, y, z, w) \mid F(x, y, z, w)=0\}
$$

Suitable implicit varieties $\left\{(x, y, z, w) \mid D_{i}(x, y, z, w)=0\right\}$ for the boundary space curves $\mathcal{C}_{i}, i=1, \ldots, 4$ where

$$
\begin{array}{ll}
\mathcal{C}_{1}(t)=\mathcal{P}(0, t), & \mathcal{C}_{2}(t)=\mathcal{P}(1, t), \\
\mathcal{C}_{3}(s)=\mathcal{P}(s, 0), & \mathcal{C}_{4}(s)=\mathcal{P}(s, 1),
\end{array}
$$

are also needed to determine the boundary curves.

Our goal is to give a semi-algebraic set to restrict the surface patch by surfaces induced by the boundary curves.

$$
S_{a}=\left\{(x, y, z, w) \mid F(x, y, z, w)=0, D_{i}(x, y, z, w) \geq 0, i=1, \ldots, 4 ; F_{j}(x, y, z, w)>0, j=1,2\right\}
$$

where $D_{i}, i=1, \ldots, 4$ are implicit equations of those cylindrical surfaces constructed for the curve $C_{i}$ in (2) by setting

$$
\mathcal{D}_{i}(u, v)=\mathcal{C}_{i}(u)+v \mathbf{v}_{i},
$$

with the new parameter $v$ and the given direction vectors $\mathbf{v}_{i}, i=1, \ldots, 4$. Note that we can easily implicitize those cylindrical surfaces by computing univariate resultants (Shen and Yuan, 2010).

The functions $F_{j}$ represent two surfaces such that

$$
\left\{(x, y, z, w) \mid D_{i}(x, y, z, w) \geq 0, i=1, \ldots, 4 ; F_{j}(x, y, z, w)>0, j=1,2\right\}
$$

forms a semi-algebraic set included in a finite volume. 


\subsection{Constructing a semi-algebraic set for planar boundary curves}

For a boundary curve $\mathcal{C}$, we first determine whether it is a planar curve. Consider a rational space curve $\mathcal{C}(t)=\left(c_{1}(t), c_{2}(t), c_{3}(t), c_{4}(t)\right)$ with degree $m$. We can write the parametrization in matrix form

$$
\mathcal{C}(t)=\left(1, t, \ldots, t^{m}\right)\left(\begin{array}{llll}
c_{10} & c_{11} & \ldots & c_{1 m} \\
c_{20} & c_{21} & \ldots & c_{2 m} \\
c_{30} & c_{31} & \ldots & c_{3 m} \\
c_{40} & c_{41} & \ldots & c_{4 m}
\end{array}\right)^{T}=\left(1, t, \ldots, t^{m}\right) \cdot M_{\mathcal{C}}
$$

The rational space curve $\mathcal{C}(t)$ is planar if and only if $\operatorname{Rank}\left(M_{\mathcal{C}}\right) \leq 3$. One can always find a plane $x_{0} x+$ $y_{0} y+z_{0} z+w_{0} w=0$ where $M_{\mathcal{C}} \cdot\left(x_{0}, y_{0}, z_{0}, w_{0}\right)=0$ if the space curve is planar. For instance, the patches of the Utah teapot have planar boundaries (see Figure 4).
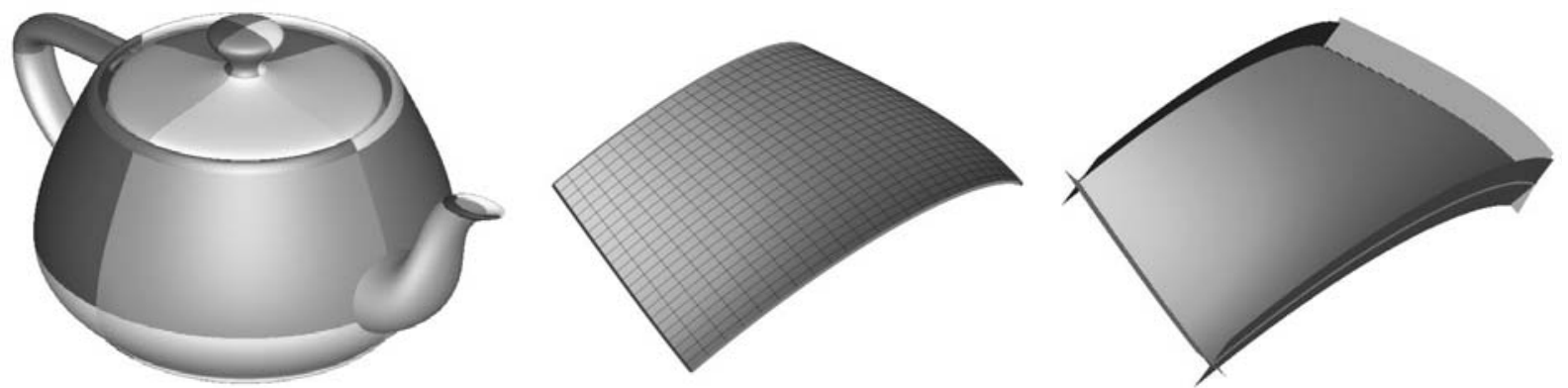

Figure 4: A surface patch of the Uath teapot with boundary planes

Here we construct two boundary surfaces $F_{i}, i=1,2$ for the surface patch. For the surface $S=$ $\{(x, y, z, w) \mid F(x, y, z, w)=0\}$, suppose there are no singular points in $S_{p}$ and there exists a point $\mathbf{p}_{0} \in S_{p}$ whose normal vector $\mathbf{v}_{0}=\mathbf{n}_{\mathbf{p}_{0}}$ in such that $\mathbf{n}_{\mathbf{p}_{0}} \cdot \mathbf{n}=\mathbf{v}_{0} \cdot\left(\frac{\partial \mathcal{P}}{\partial s} \times \frac{\partial \mathcal{P}}{\partial t}\right)$ has no real roots $(s, t) \in[0,1]^{2}$, where $\mathbf{n}_{\mathbf{p}_{0}}$ is a normal vector such that $\mathbf{n}_{\mathbf{p}_{0}}$ is not orthogonal to any other normal vectors of the points on the surface patch $S_{p}$. Moreover, there is an $\varepsilon>0$ such that the planes $F_{i}=\left((x, y, z, w)-\mathbf{p}_{0}+(-1)^{i} \varepsilon \mathbf{n}_{\mathbf{p}_{0}}\right) \cdot \mathbf{n}_{\mathbf{p}_{0}}=0, i=1,2$ have no intersections with the surface patch $S_{p}$. The condition $\left\{(x, y, z, w) \mid F_{j}=0\right\} \cap S_{p}=\emptyset$ is equivalent to the fact that there is no parameter pair $\left\{(s, t) \mid F_{j}(\mathcal{P}(s, t))=0,(s, t) \in[0,1]^{2}\right\}$. Now introduce the semi-algebraic set

$$
S_{a}=\left\{(x, y, z, w) \mid F(x, y, z, w)=0, D_{i}(x, y, z, w) \geq 0, i=1, \ldots, 4 ; F_{j}(x, y, z, w)>0, j=1,2 .\right\}
$$

The boundary planes $S_{D_{i}}$ and $S_{F_{j}}$, defined by $\mathcal{D}_{i}$ and $F_{j}$, form a hexahedron and each face of the hexahedron can be represented by a plane (see Figure 5). Hence $S_{D_{i}}$ and $S_{F_{j}}$ can each be generated by four coplanar points; for instance, $S_{D_{i}}$ is generated by the four vertex points of the quadrilateral $\mathbf{p}_{i 1}, \mathbf{p}_{i 2}, \mathbf{p}_{i 3}, \mathbf{p}_{i 4}$. Now we can easily find a rational parametrization of the plane $S_{D_{i}}$ by setting

$$
\mathcal{D}_{i}(u, v)=(1-u)\left((1-v) \mathbf{p}_{i 1}+v \mathbf{p}_{i 2}\right)+u\left((1-v) \mathbf{p}_{i 3}+v \mathbf{p}_{i 4}\right) .
$$

Note that $\mathcal{D}_{i}(u, v)$ can be an improper rational parametrization of $S_{D_{i}}$, so each point of the quadrilateral face (patch) associated to $S_{D_{i}}$ is

$$
S_{D_{i}, p}=\left\{(x, y, z, w) \mid(x, y, z, w)=\mathcal{D}_{i}(u, v), u \in[0,1], v \in[0,1]\right\} .
$$

which may have more than one parameter corresponding to each point. Similar constructions apply for the rational parametrizations $\mathcal{F}_{j}(u, v)$ of the boundary surface $S_{F_{j}}$ and the quadrilateral face (patch) is denoted by $S_{F_{i}, p}$.

When we discuss whether there are intersections between the quadrilateral face associated to $S_{D_{i}}$ and the corresponding surface $S=\{(x, y, z, w) \mid F(x, y, z, w)=0\}$, we shall invoke the following lemma. 
Lemma 2. Let $\mathcal{D}_{i}(u, v)$ be a (improper) rational parametrization of a plane $S_{D_{i}}$ and let $S_{D_{i}, p}=\{(x, y, z, w) \mid$ $(x, y, z, w)=\mathcal{D}_{i}(u, v), u \in[0,1], v \in[0,1]$ be a quadrilateral face in $S_{D_{i}}$. Then a surface $S=\{(x, y, z, w) \mid$ $F(x, y, z, w)=0\}$ has real intersections with the quadrilateral face $S_{D_{i}, p}$ if and only if $F\left(\mathcal{D}_{i}(u, v)\right)=0$ has real solutions in $(u, v) \in[0,1]^{2}$.

Proof. Necessity is obvious whether or not the rational parametrization is proper.

Sufficiency is also correct if the rational parametrization is proper. Now suppose that the parametrization $\mathcal{D}_{i}(u, v)$ is improper and there exists an intersection point $\left(x_{0}, y_{0}, z_{0}, w_{0}\right)$ belonging to $S_{D_{i}}$ in the quadrilateral face. Then there must exist at least a corresponding parameter pair $\left(u_{0}, v_{0}\right) \in[0,1]^{2}$ such that $\mathcal{D}_{i}\left(u_{0}, v_{0}\right)=$ $\left(x_{0}, y_{0}, z_{0}, w_{0}\right)$, since $\left(x_{0}, y_{0}, z_{0}, w_{0}\right)$ belongs to $S_{D_{i}, p}$. Here $\left(u_{0}, v_{0}\right) \in[0,1]^{2}$ is a real solution of $F\left(\mathcal{D}_{i}(u, v)\right)=$ 0 .
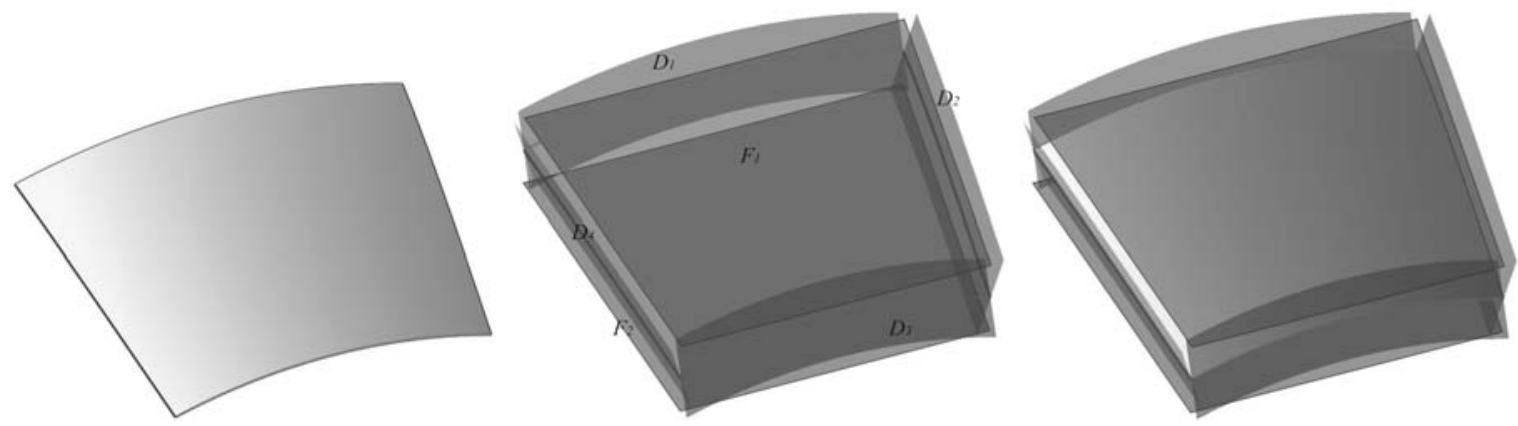

Figure 5: A surface patch included in a semi-algebraic set bounded by a hexahedron

Proposition 3. Let $S_{p}=\{(x, y, z, w) \mid(x, y, z, w)=\mathcal{P}(s, t), s \in[0,1], t \in[0,1]\}$ be a rational surface patch and let $F_{i}=\left((x, y, z, w)-\mathbf{p}_{0}+(-1)^{i} \varepsilon \mathbf{n}_{\mathbf{p}_{0}}\right) \cdot \mathbf{v}_{0}=0, i=1,2$ be two planes induced by $\mathcal{P}$. If

1) There are no singular points in $S_{p}$;

2) $\mathcal{P}\left(s_{0}, t\right), s_{0}=0,1$ and $\mathcal{P}\left(s, t_{0}\right), t_{0}=0,1$ are planar curves;

3) Each boundary plane $S_{D_{i}}$ and $S_{p}$ have no tangent point at $C_{i}$;

4) Each boundary plane $S_{D_{i}}$ and $S_{p}$ have no intersection points other than $C_{i}$;

5) Each boundary plane $S_{F_{j}}$ and $S_{p}$ have no intersection point;

6) Each boundary quadrilateral face $S_{D_{i}, p}$ and the surface $S$ have no intersection points other than $C_{i}$;

7) Each boundary quadrilateral face $S_{F_{j}, p}$ and the surface $S$ have no intersection point,

then the semi-algebraic set $S_{a}$ defined in Equation (3) is equivalent to the surface patch $S_{p}$.

Proof. According to the construction, $S_{p} \subset\left\{(x, y, z, w) \mid D_{i}(x, y, z, w) \geq 0\right\}$ and $S_{p} \subset\{(x, y, z, w) \mid$ $\left.F_{i}(x, y, z, w) \geq 0\right\}$. By conditions 4$)$ and 5), all points of the surface patch $S_{p}$ lie in the same real region determined by the boundary planes; hence $S_{p} \subset S_{a}$.

Now we show $S_{a} \subset S_{p}$. Otherwise, if there is one point $\mathbf{p}_{0} \in S_{a} \backslash S_{p}$ and another point $\mathbf{p}_{1} \in S_{p}$, then there is a path $\widetilde{\mathbf{p}_{0} \mathbf{p}_{1}} \in S$ connecting $\mathbf{p}_{0}$ and $\mathbf{p}_{1}$ since $S$ is a rational surface. The path curve $\widetilde{\mathbf{p}_{0} \mathbf{p}_{1}}$ is included in $S_{a}$ otherwise $\widetilde{\mathbf{p}_{0} \mathbf{p}_{1}}$ has intersections with at least one quadrilateral face $S_{D_{i}, p}$ or $S_{F_{j}, p}$, i.e., there are intersection points of the surface $S$ and the quadrilateral face, which cannot happen by conditions 6 ) and 7). From condition 3$), \mathbf{p}_{1}$ connects to $\mathbf{p}_{0}$ without touching any point at $C_{i}$ on the boundary planes $S_{D_{i}}$. Thus $\widetilde{\mathbf{p}_{0} \mathbf{p}_{1}}$ must leave $S_{p}$ at a certain point $\mathbf{p}_{m}$ and $\mathbf{p}_{m}$ is a singular point, which cannot happen by condition 1). Therefore there is no such point $\mathbf{p}_{0}$ so $S_{a}=S_{p}$. 
The main task is to check the conditions in Proposition 3.

1. This condition means that $\frac{\partial F}{\partial x}=\frac{\partial F}{\partial y}=\frac{\partial F}{\partial z}=\frac{\partial F}{\partial w}=0$ has no point in $S_{p}$. Hence this condition is equivalent to the set $\frac{\partial F}{\partial x}(\mathcal{P})=\frac{\partial F}{\partial y}(\mathcal{P})=\frac{\partial F}{\partial z}(\mathcal{P})=\frac{\partial F}{\partial w}(\mathcal{P})=0$ having no real solutions $(s, t) \in[0,1]^{2}$;

2. This condition is discussed at the start of Section 3.1;

3. The normal vector along the curve $C_{1}$ is $\left.\left(\frac{\partial \mathcal{P}}{\partial s} \times \frac{\partial \mathcal{P}}{\partial t}\right)\right|_{s=0}$. This condition is equivalent to $\left.\left(\frac{\partial \mathcal{P}}{\partial s} \times \frac{\partial \mathcal{P}}{\partial t}\right)\right|_{s=0}=$ $\left(x_{0}, y_{0}, z_{0}, w_{0}\right)$ has no real solutions $t \in[0,1]$ where $\left(x_{0}, y_{0}, z_{0}, w_{0}\right)$ is the normal vector of $D_{1}$. Similar remarks apply to $D_{i}, i=2,3,4$;

4. Substituting $\mathcal{P}(s, t)$ into the implicit equation $D_{1}(x, y, z, w)=0$, we get the equation $D_{1}(s, t)=$ $D_{1}(\mathcal{P}(s, t))=0$. Since the curve $\mathcal{C}_{1}(t)$ belongs to the intersection, $s$ is a factor of $D_{1}(s, t)$. Now check $D_{1}(s, t) / s=0$ as a planar curve in $(s, t)$-space; if there is no real part belonging to $\{(s, t) \mid s \in[0,1], t \in$ $[0,1]\}$ then the plane $S_{D_{1}}$ is a good boundary plane associated to the surface patch. Here, good is equivalent to the fact that the surface patch $S_{p}$ lies only on one of the half spaces separated by the boundary plane. Similar remarks apply to $D_{i}, i=2,3,4$;

5. $F_{j}(\mathcal{P}(s, t))=0$ have no real part belonging to $\{(s, t) \mid s \in[0,1], t \in[0,1]\}$;

6. $F\left(\mathcal{D}_{i}(u, v)\right)=0$ have no real part belonging to $\{(u, v) \mid u \in[0,1], v \in[0,1]\}$ other than the curve $D_{i}$. To make the curve $\mathcal{C}_{i}$ be the boundary curve, we replace $D_{i}$ by two patches

$$
\begin{aligned}
& \mathcal{D}_{i 1}(u, v)=u\left((1-v) \mathbf{p}_{i 1}+v \mathbf{p}_{i 2}\right)+(1-u) C_{i}(v) ; \\
& \mathcal{D}_{i 2}(u, v)=u\left((1-v) \mathbf{p}_{i 3}+v \mathbf{p}_{i 4}\right)+(1-u) C_{i}(v)
\end{aligned}
$$

where the curve $\mathcal{C}_{i}$ is from (2).

Since the curve $\mathcal{C}_{i}(v)$ belongs to the intersection, $u$ is a factor of $F(s, t)=F\left(\mathcal{D}_{i j}(u, v)\right)$. Now check if $f_{j}(u, v)=F\left(\mathcal{D}_{i j}(u, v)\right) / u=0, j=1,2$ as a planar curve in $(u, v)$-space; if there is no real part belonging to $\{(u, v) \mid s \in[0,1], t \in[0,1]\}$ for $f_{j}=0, j=1,2$ then the plane $D_{i}$ satisfies condition 6$)$.

7. $F\left(\mathcal{F}_{j}(u, v)\right)=0$ have no real part belonging to $\{(u, v) \mid u \in[0,1], v \in[0,1]\}$

Proposition 4. Let $S_{p}=\{(x, y, z, w) \mid(x, y, z, w)=\mathcal{P}(s, t), s \in[0,1], t \in[0,1]\}$ be a rational surface patch. If

1) there are no singular points in $S_{p}$;

2) for any fixed $s_{0} \in[0,1]$ and $t_{0} \in[0,1]$ the space curves $\mathcal{P}\left(s_{0}, t\right)$ and $\mathcal{P}\left(s, t_{0}\right)$ are planar curves;

3) the intersection curve of each plane from 2) and $S_{p}$ have no tangent points along the corresponding curve segment;

4) the intersection curve of each plane from 2) and $S_{p}$ have no singular points.

then this surface patch can be represented by the union of semi-algebraic sets

$$
\begin{array}{r}
\bigcup_{k=1}^{m} S_{a, k}=\bigcup_{k=1}^{m}\left\{(x, y, z, w) \mid F(x, y, z, w)=0, D_{k i}(x, y, z, w) \geq 0, i=1, \ldots, 4 ;\right. \\
\left.F_{k j}(x, y, z, w)>0, j=1,2 .\right\}
\end{array}
$$

Proof. For a surface patch $S_{p}$, conditions 1)-3) are similar to Proposition 3. Suppose that some of conditions 4)-7) are not satisfied, we will subdivide the Bézier patch to four smaller Bézier patches. If conditions 4)-7) on each patch hold, then we complete the construction. Otherwise, we continue to subdivide the patch on which some of conditions 4)-7) are not satisfied.

We now show that each of conditions 4)-7) will hold on the subdivided patches after a finite number of subdivision steps.

Since there is no singular point in $S_{p}$, for a point $\mathbf{p}_{0}$ associated to $\left(s_{0}, t_{0}\right)$, there is an $\epsilon>0$ and $\varepsilon_{\epsilon}$ for the constructed semi-algebraic set for the patch $S_{p, \epsilon}$ with $s \in\left[s_{0}-\epsilon, s_{0}+\epsilon\right], t \in\left[t_{0}-\epsilon, t_{0}+\epsilon\right]$ such that the $F_{i}=\left((x, y, z, w)-\mathbf{p}_{0}+(-1)^{i} \varepsilon_{\epsilon} \mathbf{n}_{\mathbf{p}_{0}}\right) \cdot \mathbf{n}_{\mathbf{p}_{0}}=0, i=1,2$ satisfy conditions 5$)$ and 7$)$ of Proposition 3. Note that $F_{i}$ are simply generated as offset planes of the tangent plane at $\mathbf{p}_{0}$, but the planes are not necessarily offsets. 
Now we check conditions 4) and 6) of Proposition 3 for the boundary planes $S_{D_{i}}$ associated to the patch $S_{p, \epsilon}$. For condition 6), if the quadrilateral face $S_{D_{1}, p}$ and the surface $S$ have intersections other than the boundary curve segment $C_{1}$, the other intersections are separated from $C_{1}$ since the intersections cannot be singular by assumption 4). Then we can decrease $\epsilon$ and $\varepsilon_{\epsilon}$ so that the quadrilateral face $S_{D_{1}, p}$ and the surface $S$ have no intersections other than the boundary curve segment $C_{1}$. Similar modifications apply for $D_{i}$ such that condition 6) of Proposition 3 holds.

Finally, if 4) of Proposition 3 does not hold, then $S_{D_{1}}$ and $S_{p, \epsilon}$ have intersections other than the boundary curve segment $C_{1}$ but separated from $C_{1}$ since $S_{p, \epsilon}$ have no singular points. Thus there exists $\tilde{s}_{0} \in\left[s_{0}-\right.$ $\left.\epsilon, s_{0}+\epsilon\right]$ such that a curve segment $\mathcal{P}\left(s_{0}, t\right), t \in\left[t_{0}-\epsilon, t_{0}+\epsilon\right]$ has no points belonging to $S_{D_{1}} \cap S_{p, \epsilon}$. Subdivide $S_{p, \epsilon}$ at $\tilde{s}_{0}$ into $S_{p, \epsilon, l}$ and $S_{p, \epsilon, r}$; then condition 4) of Proposition 3 holds for $S_{D_{1}}$ with respect to $S_{p, \epsilon, l}$. Suppose this condition does not hold for $S_{p, \epsilon, r}$. Then we can use a similar subdivision process and the process will terminate in a finite number of steps since the intersection curve of each plane from 2) and $S_{p}$ has no singular points. Similar remarks apply for the three boundary planes. Now there is a subdivided patch including $\mathbf{p}_{0}$ such that 4 ) of Proposition 3 holds on this patch.

Thus, we get a patch whose semi-algebraic set satisfies all the conditions of Proposition 3. Till now, we find a neighbour semi-algebraic set for a point $\mathbf{p}_{0} \in S_{p}$. But after certain subdivisions, we can finally get a union of semi-algebraic sets that covers the patch $S_{p}$.

\subsection{Constructing a semi-algebraic set for general boundary curves}

A patch with planar boundaries can have a semi-algebraic set included in simple hexahedron, but most patches may have nonplanar boundaries. For the general patches whose boundary space curves $\mathcal{C}(t)$ are not planar, we need to introduce the boundary surfaces associated to the boundary curves by constructing cylindrical surfaces.

For the surface $S=\{(x, y, z, w) \mid F(x, y, z, w)=0\}$, suppose there are no singular points in $S_{p}$ and there exists a vector $\mathbf{v}_{0}$ such that $\mathbf{v}_{0} \cdot\left(\frac{\partial \mathcal{P}}{\partial s} \times \frac{\partial \mathcal{P}}{\partial t}\right)$ has no real solutions $(s, t) \in[0,1]^{2}$. Then there is an $\varepsilon>0$ such that $\mathcal{P}_{i}(s, t)=\mathcal{P}(s, t)+(-1)^{i} \varepsilon \mathbf{v}_{0}, i=1,2$ has no intersections with the surface patch $S_{p}$. Let $F_{i}=0$ be the implicit equation of $\mathcal{P}_{i}(s, t)$, which is given explicitly by

$$
F_{i}(x, y, z, w)=F\left(x+(-1)^{i} \varepsilon v_{1}, y+(-1)^{i} \varepsilon v_{2}, z+(-1)^{i} \varepsilon v_{3}, w+(-1)^{i} \varepsilon v_{4}\right)
$$

with $\mathbf{v}_{0}=\left(v_{1}, v_{2}, v_{3}, v_{4}\right)$. Then $\left\{(x, y, z, w) \mid F_{i}=0\right\} \cap S_{p}=\emptyset$ is equivalent to the fact that there is no real parameter $\left\{(s, t) \mid F_{j}(\mathcal{P}(s, t))=0,(s, t) \in[0,1]^{2}\right\}$. To construct the cylindrical surfaces, we select the vectors $\mathbf{v}_{1}=\mathbf{v}_{2}=\mathbf{v}_{3}=\mathbf{v}_{4}=\mathbf{v}_{0}$ (see Figure 6). Then $\mathcal{D}_{1}(t, u)=\mathcal{C}_{1}(t)+\mathbf{v}_{0} u, \mathcal{D}_{2}(t, u)=\mathcal{C}_{2}(t)+\mathbf{v}_{0} u$,
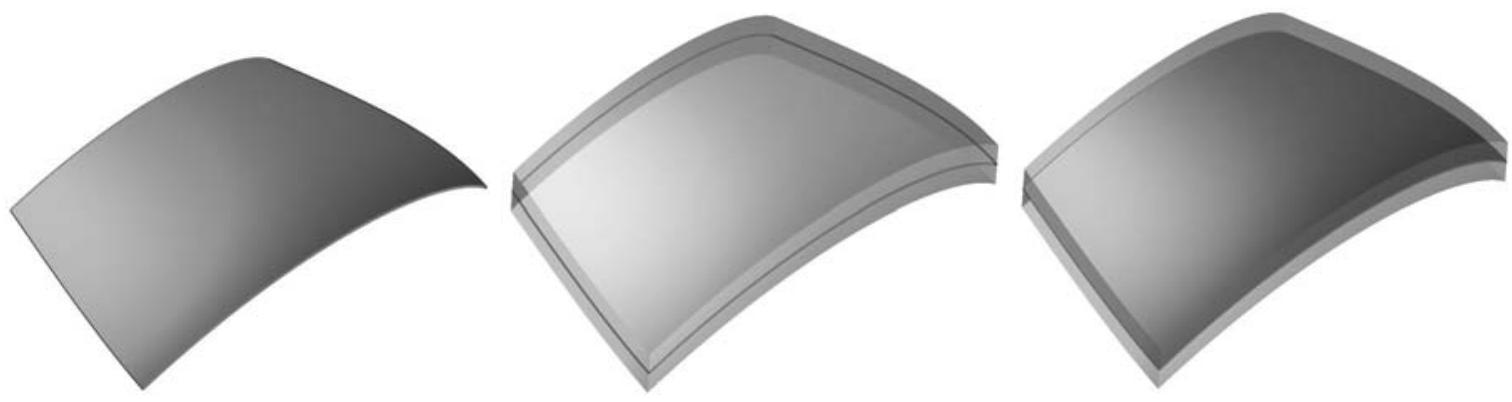

Figure 6: A surface patch included in a semi-algebraic set having four boundary cylindrical surfaces

$\mathcal{D}_{3}(t, u)=\mathcal{C}_{3}(s)+\mathbf{v}_{0} u, \mathcal{D}_{4}(t, u)=\mathcal{C}_{4}(s)+\mathbf{v}_{0} u$, are rational parametrizations of the boundary cylindrical surfaces. Now the semi-algebraic set

$$
S_{a}=\left\{(x, y, z, w) \mid F(x, y, z, w)=0, D_{i}(x, y, z, w) \geq 0, i=1, \ldots, 4 ; F_{j}(x, y, z, w)>0, j=1,2 .\right\}
$$

is a part of a column generated by the surface patch $P$ and the vector $\mathbf{v}_{0}$. 
Proposition 5. Let $S_{p}=\{(x, y, z, w) \mid(x, y, z, w)=\mathcal{P}(s, t), s \in[0,1], t \in[0,1]\}$ be a rational surface patch, and let $F_{j}(x, y, z, w)=F\left(x+(-1)^{i} \varepsilon v_{1}, y+(-1)^{i} \varepsilon v_{2}, z+(-1)^{i} \varepsilon v_{3}, w+(-1)^{i} \varepsilon v_{4}\right)$ be two surfaces induced by $S_{p}$ where $\mathbf{v}_{0}=\left(v_{1}, v_{2}, v_{3}, v_{4}\right)$ is a vector $\mathbf{v}_{0}$ such that $\mathbf{v}_{0} \cdot\left(\frac{\partial \mathcal{P}}{\partial s} \times \frac{\partial \mathcal{P}}{\partial t}\right)$ has no real solutions $(s, t) \in[0,1]^{2}$. If

1) there are no singular points in $S_{p}$;

2) Each bounding cylinder $D_{i}$ and $S_{p}$ have no tangent point at $C_{i}$;

3) Each bounding cylinder $D_{i}$ and $S_{p}$ have no intersection point other than $C_{i}$;

4) Each boundary surface $F_{i}$ and $S_{p}$ have no intersection point;

5) Each boundary cylindrical surface patch $S_{D_{i}, p}=\left\{(x, y, z, w) \mid(x, y, z, w)=\mathcal{C}_{i}(u)+v \mathbf{v}_{0}, v \in[-\varepsilon, \varepsilon], u \in\right.$ $[0,1]\}$ and the surface $S$ have no intersection point other than $C_{i}$;

6) Each boundary surface patch $S_{F_{j}, p}=\left\{(x, y, z, w) \mid(x, y, z, w)=\mathcal{P}(s, t)+(-1)^{i} \varepsilon \mathbf{v}, i=1,2, s \in[0,1], t \in\right.$ $[0,1]\}$ and the surface $S$ have no intersection point,

then the semi-algebraic set $S_{a}$ in Equation (4) is equivalent to the surface patch $S_{p}$.

Proof. The proof of this result is similar to the proof of Proposition 3.

For all the surfaces $S_{D_{i}}$ and $S_{F_{j}}$, their parametrization and implicit equations are given explicitly. The parametrizations $S_{D_{i}}$ may be improper, but we can still proceed by invoking Lemma 2. Once we get the semi-algebraic set $S_{a}$, we can also find, whenever needed, a proper parametrization for $S_{D_{i}}$ (Li et al., 2008).

Proposition 6. Let $S_{p}=\{(x, y, z, w) \mid(x, y, z, w)=\mathcal{P}(s, t), s \in[0,1], t \in[0,1]\}$ be a rational surface patch. For a general vector $\mathbf{v}$, if

1) there are no singular points in $S_{p}$;

2) the intersection curve of each cylindrical surface $S_{D_{i}}$ and $S_{p}$ have no tangent points along the corresponding curve segment;

3) the intersection curve of each cylindrical surface $S_{D_{i}}$ and $S_{p}$ has no singular points.

then this surface patch can be represented by the union of semi-algebraic sets

$$
\begin{array}{r}
\bigcup_{k=1}^{m} S_{a, k}=\bigcup_{k=1}^{m}\left\{(x, y, z, w) \mid F(x, y, z, w)=0, D_{k i}(x, y, z, w) \geq 0, i=1, \ldots, 4 ;\right. \\
\left.F_{k j}(x, y, z, w)>0, j=1,2 .\right\}
\end{array}
$$

Proof. The proof of this result is similar to the proof of Proposition 4.

\subsection{Real Root Determination}

We need to compute the real intersection of two boundary surfaces in the construction of a semi-algebraic set $S_{a}$. This problem is equivalent to determining the existence of real roots of a planar curve within a given parameter region. Precisely, for a planar curve $f(s, t)=0$, we will determine the existence of real components in $[0,1]^{2}$. Several standard methods can be used to determine these real components such as Cylindrical Algebraic Decomposition, solving the polynomial system (Mourrain and Pavone, 2009; Barton̆ et al., 2011) and topology analysis of planar curves (Eigenwillig et al., 2007; Cheng et al., 2009; Zapata and Martín, 2014). Here we would like to give a simple approach to solving this problem. We proceed in the following manner: Compute the real roots of the following system

$$
\left\{\begin{array}{l}
\operatorname{Res}_{t}\left(f(s, t), \frac{\partial f}{\partial t}(s, t)\right)=0 \\
\operatorname{Res}_{t}\left(f(s, t), \frac{\partial f}{\partial s}(s, t)\right)=0 \\
f(s, 0)=0 \\
f(s, 1)=0
\end{array}\right.
$$


Suppose that these roots are $s_{1}<s_{2}<\cdots<s_{k}$. Then the curve segments in each interval $\left[s_{i}, s_{i+1}\right]$ are monotone, i.e., the product $\frac{\partial f}{\partial s}(s, t) \frac{\partial f}{\partial t}(s, t)$ in $\left[s_{i}, s_{i+1}\right]$ does not change sign. Select $0=s_{i_{1}}<s_{i_{2}}<\cdots<$ $s_{i_{l-1}}<s_{i_{l}}=1$ and consider $f\left(s_{i_{j}}, t\right)=0$ where $s_{i_{j}} \in\left\{s_{1}, \cdots, s_{k}\right\}, j=2, \ldots, l-1$. Then $f(s, t)=0$ has real parts in $[0,1]^{2}$ if $f\left(s_{i_{j}}, t\right)=0$ has real segments in $[0,1]$ for any $j \in\{1, \cdots, l\}$.

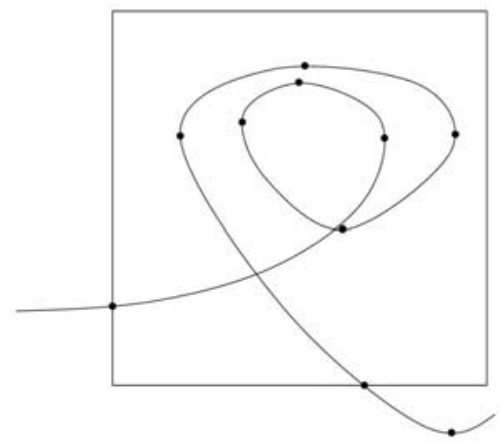

Figure 7: Real part determination

\subsection{Implicitization Algorithm of Surface Patches}

Lemma 3. For a rational surface patch $\mathcal{P}(s, t), s \in[0,1], t \in[0,1]$ and a vector $\mathbf{v}_{0}$, the surface patches $S_{F_{i}, p}$ and $S_{p}$ have no intersection point if the unit normal vectors of the patch are strictly included in half a unit sphere and $\mathbf{v}_{0}$ can be a normal vector at any point of $\mathcal{P}(s, t), s \in[0,1], t \in[0,1]$.

Proof. Let $v_{0}$ be a normal vector at a point of $\mathcal{P}(s, t), s \in[0,1], t \in[0,1]$. A line parallel to $\mathbf{v}_{0}$ has at most one real intersection point with the surface patch $S_{p}$ since the unit normal vectors of the segment are strictly included in half a sphere, i.e., the surface patch is a single-valued function relative to the vector $\mathbf{v}_{0}$. Hence, any surface patch $S_{F_{i}, p}$ along the vector $\mathbf{v}_{0}$ has no intersection with $S_{p}$.

Algorithm 3. Find an exact semi-algebraic set for a rational surface patch $\mathcal{P}(s, t), s \in[0,1], t \in[0,1]$ having no singular points.

1. Find a vector $\mathbf{v}_{0}$ satisfying Lemma 3 (and not parallel to tangent vectors of the surface patch at the boundary curves). If no such vector $\mathbf{v}_{0}$ exists, subdivide the surface patch and such that there exist such $\mathbf{v}_{0}$ satisfying Lemma 3 for each subdivided segment.

2. Construct an initial semi-algebraic set for the curve segments by the above set of the form $S_{a}$.

3. Check conditions 2)-6) of Proposition 5 and update $\varepsilon=\varepsilon / 2$ if some conditions do not hold.

Surface patches without singular points are popular in geometric modeling and there are methods to design surface patches without singular points by checking certain geometric conditions (Zhao and Zhu, 2015; Zhao et al., 2017). For a surface patch without singular points, we can find a semi-algebraic set for the patch.

For the case where there might be singular points in the surface patch, we need to detect the existence of singular points. Since singularity analysis for rational surfaces is an important problem, there are many papers on computing these singularities including symbolic, numeric and hybrid techniques (Krishnan and Manocha, 1997; Andersson et al., 1998; Chen et al., 2006; Galligo and Pavone, 2006; Elber et al., 2009; Shen et al., 2012; Pérez-Díaz et al., 2015). Therefore this topic is another typical problem in surfaces modeling and we shall assume there are techniques which can be used to deal with this problem.

Algorithm 4. Find a semi-algebraic set for a rational surface patch $\mathcal{P}(s, t), s \in[0,1], t \in[0,1]$ with given error.

1. Compute the implicit equation $F(x, y, z, w)$ of $\mathcal{P}(s, t)$; 
2. Check whether there exist singular points on the patch. If there are singular points and the patch is small enough, approximate the patch using some typical methods introduced above, otherwise subdivide the patch.

3. For a surface patch with no singular points, find an exact semi-algebraic set using Algorithm 3.

\section{Experiments}

We have implemented our algorithm to find the semi-algebraic sets for the patches of the Utah teapot (see Figure 4, http://www.holmes3d.net/graphics/teapot/). This teapot is formed from 32 bicubic Bézier surface patches. Removing symmetric cases, we are left with ten different types of patches: upper lid, lower lid, upper body, middle body, lower body, upper spout, lower spout, upper handle, lower handle, bottom. We find that none of these patches have singular points and so we succeed to compute the semi-algebraic set for each patch. We display these patches, their semi-algebraic sets and their composites in Figures 8 and 9. Some patches need to be subdivided into smaller pieces. All the semi-algebraic sets and the teapot are displayed in Figure 10.

Our algorithm runs with Maplesoft 2017 on an Acer Swift5 Ultrabook with RAM 8G and Intel(R) Core(TM)@1.8 GHz, OS-Windows 10. The most time consuming parts of our algorithm deal with implicitizing the surfaces, finding the direction vector $\mathbf{v}_{0}$, and detecting the real roots in verifying the conditions of Proposition 5. For implicitization, the timings are listed in (Shen and Goldman, 2017, 2018). After our construction, we have both implicit and parametric expressions for the boundary surfaces. Thus detecting real roots for verifying the conditions in Proposition 5 are quick (about two-three CPU seconds). The time required to find the correct vector $\mathbf{v}_{0}$ has some randomness. Here we find the vector $\mathbf{v}_{0}$ by testing the vectors in the half sphere given by Lemma 3. We can often get the correct vector quickly, but the worst case may cost about 10 minutes to get the correct vector or to subdivide the patch. Note that, in general, more subdivisions for the patch make finding $\mathbf{v}_{0}$ easier.

\section{Conclusion}

The representation of curve segments and surface patches are basic tasks in geometric modeling and computer graphics. The parametric form and mesh generation are commonly used. But for exact or highly precise computations one may need to introduce a large amount of parametric data or mesh segments in some regions with detailed features. This paper proposes an alternative framework, expressing curve segments and surface patches by semi-algebraic sets, which can be used as implicit representations. Both implicit equations and parametric expressions are given for the bounding curves/surfaces of the semi-algebraic set.

For a surface patch with singular points; suppose the singularities are known; if we want to approximate more details such that singularities are preserved, then subtle topological analysis is needed. Based on this analysis, one way to represent a surface patch with singularities is to approximate the given surface patch with new surface patches. These approximate surface patches have no singular points but we can recover the singular points of the given patch from the intersection points of these approximating patches. We hope to provide such a subtle analysis in our future work.

\section{Acknowledgement}

This work has been partially funded by National Natural Science Foundation of China under Grant 61872332, 11731013 and by the Spanish Ministerio de Ciencia, Innovación y Universidades and by the European Regional Development Fund (ERDF), under the project MTM2017-88796-P. The second author belongs to the Research Group ASYNACS (Ref. CCEE2011/R34). 


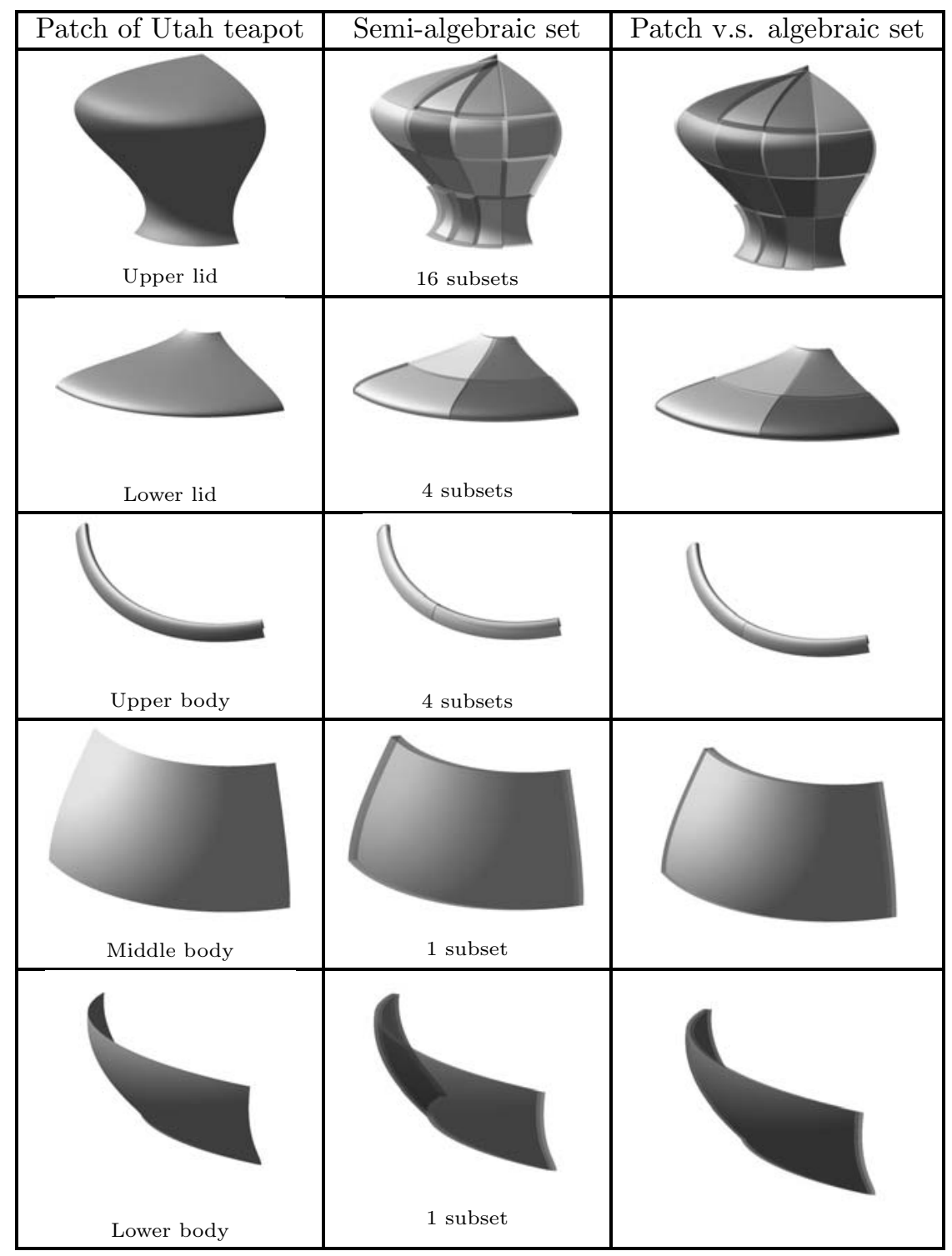

Figure 8: The patches of the Utah teapot and their semi-algebraic sets 


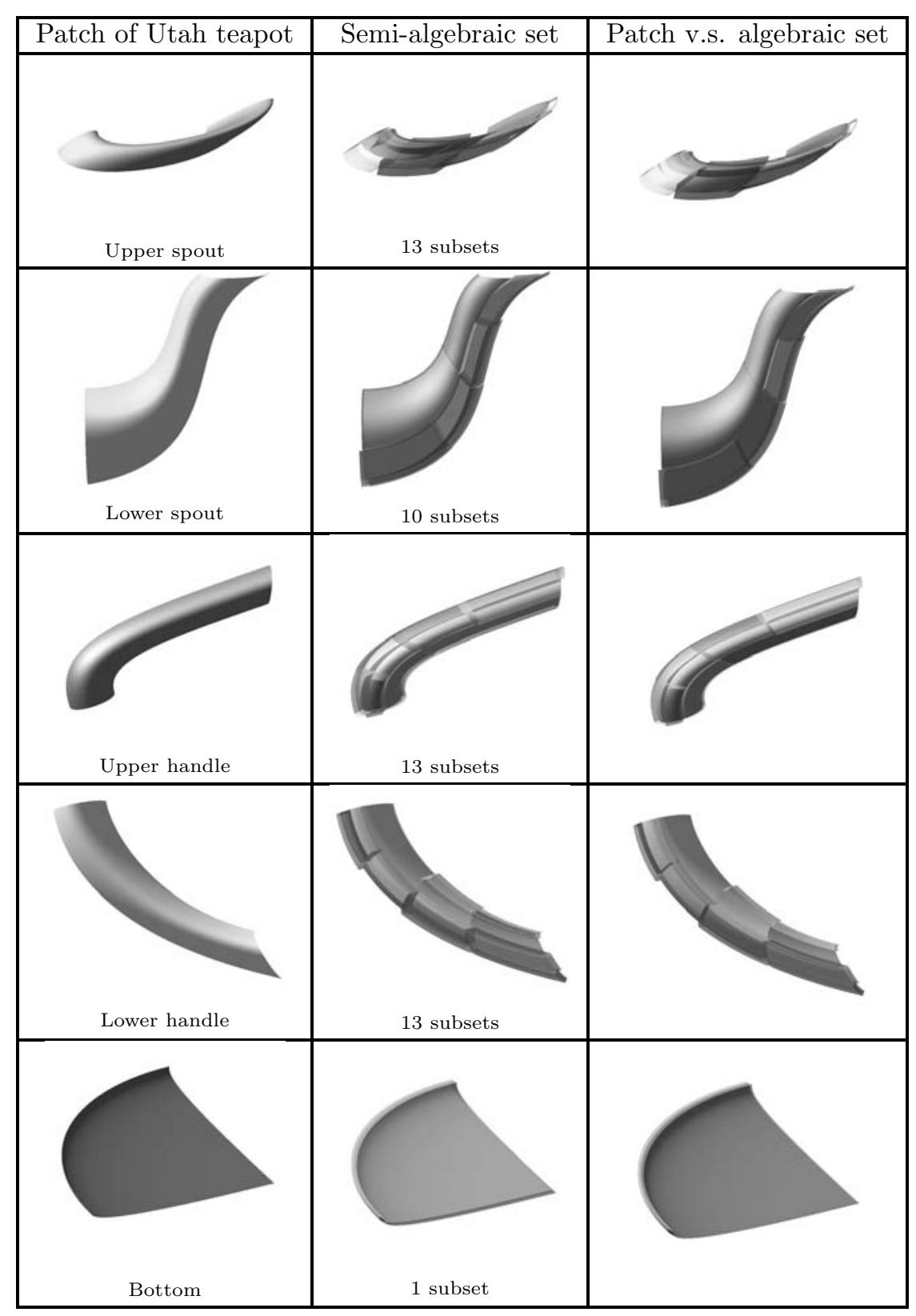

Figure 9: The patches of the Utah teapot and their semi-algebraic sets 

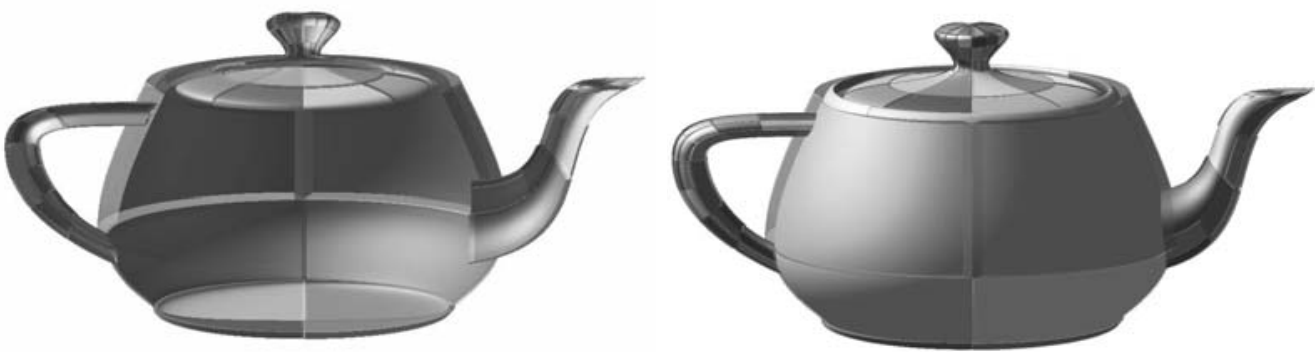

Figure 10: All the semi-algebraic sets for the teapot and these sets v.s. the teapot.

\section{References}

Andersson, L.E., Peters, T., Stewart, N., 1998. Selfintersection of composite curves and surfaces. Computer Aided Geometric Design 15, 507 - 527. URL: http://www.sciencedirect.com/science/article/pii/S0167839698000053, doi:https://doi. org/10.1016/S0167-8396(98)00005-3.

Bartoŭ, M., Elber, G., Hanniel, I., 2011. Topologically guaranteed univariate solutions of underconstrained polynomial systems via no-loop and single-component tests. Computer-Aided Design 43, 1035 - 1044. URL: http://www.sciencedirect.com/ science/article/pii/S0010448511000819, doi:https://doi.org/10.1016/j.cad.2011.03.009.

Buchberger, B., 1985. Groebner-Bases: An Algorithmic Method in Polynomial Ideal Theory, in: Bose, N. (Ed.), Multidimensional Systems Theory - Progress, Directions and Open Problems in Multidimensional Systems. Copyright: Reidel Publishing Company, Dordrecht - Boston - Lancaster, The Netherlands. chapter 6, pp. 184-232. (Second editioin: N.K.Bose (ed.): Multidimensional Systems Theory and Application, Kluwer Academic Publisher, 2003, pp.89-128.).

Buhmann, M.D., 2003. Radial basis functions: Theory and implementations. Cambridge University Press.

Busé, L., 2014. Implicit matrix representations of rational Bézier curves and surfaces. Computer-Aided Design $46,14-24$. URL: http://www.sciencedirect.com/science/article/pii/s0010448513001541, doi:http://dx.doi.org/10.1016/j.cad. 2013.08.014. 2013 \{SIAM\} Conference on Geometric and Physical Modeling.

Carr, J.C., Beatson, R.K., Cherrie, J.B., Mitchell, T.J., Fright, W.R., McCallum, B.C., Evans, T.R., 2001. Reconstruction and representation of $3 \mathrm{D}$ objects with radial basis functions, in: Proceedings of the 28th Annual Conference on Computer Graphics and Interactive Techniques, ACM, New York, NY, USA. pp. 67-76. URL: http://doi.acm.org/10.1145/383259. 383266, doi:10.1145/383259.383266.

Caviness, B.F., Johnson, J.R., 1998. Quantifier Elimination and Cylindrical Algebraic Decomposition. Springer-Verlag Wien. doi:10.1007/978-3-7091-9459-1.

Chen, F., Cox, D.A., Liu, Y., 2005. The $\mu$-basis and implicitization of a rational parametric surface. Journal of Symbolic Computation 39, 689 - 706. URL: http://www.sciencedirect.com/science/article/pii/S0747717105000398, doi:http: //dx.doi.org/10.1016/j.jsc.2005.01.003.

Chen, F., Wang, W, 2002. The $\mu$-basis of a planar rational curve-properties and computation. Graphical Models 64, 368 - 381. URL: http://www.sciencedirect.com/science/article/pii/S1077316902000175, doi:https://doi.org/10.1016/ S1077-3169(02)00017-5.

Chen, X., Riesenfeld, R.F., Cohen, E., Damon, J., 2006. Theoretically based robust algorithms for tracking intersection curves of two deforming parametric surfaces, in: Kim, M.S., Shimada, K. (Eds.), Geometric Modeling and Processing - GMP 2006, Springer Berlin Heidelberg, Berlin, Heidelberg. pp. 101-114.

Cheng, J.S., Gao, X.S., Li, J., 2009. Topology determination and isolation for implicit plane curves, in: Proceedings of the 2009 ACM Symposium on Applied Computing, ACM, New York, NY, USA. pp. 1140-1141. URL: http://doi.acm.org/10. 1145/1529282.1529534, doi:10.1145/1529282.1529534.

Cheng, J.S., Jin, K., 2015. A generic position based method for real root isolation of zero-dimensional polynomial systems. Journal of Symbolic Computation 68, $204-224$. URL: http://www.sciencedirect.com/science/article/pii/ S0747717114000856, doi:https://doi.org/10.1016/j.jsc.2014.09.017.

Chionh, E. W., 2001. Rectangular Corner Cutting and Dixon $\mathcal{A}$-resultants. Journal of Symbolic Computation 31 , 651 669. URL: http://www.sciencedirect.com/science/article/pii/S0747717103001081, doi:https://doi.org/10.1016/j. jsc.2003.06.001.

Cohen-Or, D., Kaufman, A., 1995. Fundamentals of surface voxelization. Graphical Models and Image Processing 57, 453 - 461. URL: http://www.sciencedirect.com/science/article/pii/S1077316985710398, doi:https://doi.org/10.1006/ gmip. 1995.1039.

Eigenwillig, A., Kerber, M., Wolpert, N., 2007. Fast and exact geometric analysis of real algebraic plane curves, in: Proceedings of the 2007 International Symposium on Symbolic and Algebraic Computation, ACM, New York, NY, USA. pp. 151-158. URL: http://doi.acm.org/10.1145/1277548.1277570, doi:10.1145/1277548.1277570. 
Elber, G., Grandine, T., Kim, M.S., 2009. Surface self-intersection computation via algebraic decomposition. ComputerAided Design 41, 1060 - 1066. URL: http://www.sciencedirect.com/science/article/pii/S0010448509002280, doi:https: //doi.org/10.1016/j.cad.2009.07.008.

Elkadi, M., Mourrain, B., 2004. Residue and implicitization problem for rational surfaces. Applicable Algebra in Engineering, Communication and Computing 14, 361-379. URL: https://doi.org/10.1007/s00200-003-0139-z, doi:10.1007/ s00200-003-0139-z.

Galligo, A., Pavone, J.P., 2006. A sampling algorithm computing self-intersections of parametric surfaces, in: Elkadi, M., Mourrain, B., Piene, R. (Eds.), Algebraic Geometry and Geometric Modeling, Springer Berlin Heidelberg, Berlin, Heidelberg. pp. 185-204.

Jia, X., Goldman, R., 2009. $\mu$-bases and singularities of rational planar curves. Computer Aided Geometric Design 26, 970 - 988. URL: http://www.sciencedirect.com/science/article/pii/S016783960900079X, doi:https://doi.org/10.1016/j. cagd.2009.08.001.

Krishnan, S., Manocha, D., 1997. An efficient surface intersection algorithm based on lower-dimensional formulation. ACM Trans. Graph. 16, 74-106. URL: http://doi.acm.org/10.1145/237748.237751, doi:10.1145/237748.237751.

Laine, S., 2013. A topological approach to voxelization. Computer Graphics Forum 32, 77-86. URL: https://onlinelibrary . wiley.com/doi/10.1111/cgf.12153, doi:https://doi.org/10.1111/cgf.12153.

Li, J., Shen, L.Y., Gao, X.S., 2008. Proper reparametrization of rational ruled surface. Journal of Computer Science and Technology 23, 290-297. URL: https://doi.org/10.1007/s11390-008-9131-1, doi:10.1007/s11390-008-9131-1.

Manocha, D., Canny, J.F., 1992. Algorithm for implicitizing rational parametric surfaces. Computer Aided Geometric Design 9, 25 - 50. URL: http://www.sciencedirect.com/science/article/pii/016783969290051P, doi:http://dx.doi.org/10. 1016/0167-8396(92) 90051-P.

Mourrain, B., Pavone, J., 2009. Subdivision methods for solving polynomial equations. Journal of Symbolic Computation 44, 292 - 306. URL: http://www.sciencedirect.com/science/article/pii/S0747717108001168, doi:https://doi.org/10. 1016/j.jsc.2008.04.016. polynomial System Solving in honor of Daniel Lazard.

Pérez-Díaz, S., 2007. Computation of the singularities of parametric plane curves. Journal of Symbolic Computation 42,835 - 857. URL: http://www.sciencedirect.com/science/article/pii/s0747717107000636, doi:https://doi.org/10.1016/j. jsc.2007.06.001.

Pérez-Díaz, S., Sendra, J.R., Villarino, C., 2015. Computing the singularities of rational surfaces. Mathematics of Computation 84, 1991-2021. URL: https://doi.org/10.1090/S0025-5718-2014-02907-4, doi:https://doi.org/10.1090/ S0025-5718-2014-02907-4.

Sagraloff, M., Mehlhorn, K., 2016. Computing real roots of real polynomials. Journal of Symbolic Computation 73 , 46 - 86. URL: http://www.sciencedirect.com/science/article/pii/S0747717115000292, doi:https://doi.org/10.1016/j. jsc. 2015.03 .004

Sederberg, T.W., Chen, F., 1995. Implicitization using moving curves and surfaces, in: Proceedings of the 22Nd Annual Conference on Computer Graphics and Interactive Techniques, ACM, New York, NY, USA. pp. 301-308. URL: http: //doi.acm.org/10.1145/218380.218460, doi:http://dx.doi.org/10.1145/218380.218460.

Shen, L.Y., Cheng, J.S., Jia, X., 2012. Homeomorphic approximation of the intersection curve of two rational surfaces. Computer Aided Geometric Design 29, 613 - 625. URL: http://www.sciencedirect.com/science/article/pii/S0167839612000787, doi:https://doi.org/10.1016/j.cagd.2012.02.005.

Shen, L.Y., Goldman, R., 2017. Implicitizing rational tensor product surfaces using the resultant of three moving planes. ACM Trans. Graph. 36, 1-14. URL: http://doi.acm.org/10.1145/3119909, doi:10.1145/3119909.

Shen, L.Y., Goldman, R., 2018. Combining complementary methods for implicitizing rational tensor product surfaces. Computer-Aided Design 104, 100 - 112. URL: http://www.sciencedirect.com/science/article/pii/S0010448518304081, doi:https://doi.org/10.1016/j.cad.2018.07.002.

Shen, L.Y., Yuan, C.M., 2010. Implicitization using univariate resultants. Journal of Systems Science and Complexity 23, 804-814. URL: https://doi.org/10.1007/s11424-010-7218-6, doi:10.1007/s11424-010-7218-6.

Yao, S., Feng, Y., Jia, X., Shen, L.Y., 2019. A package to compute implicit equations for the rational curves and surfaces. in: The 44th International Symposium on Symbolic and Algebraic Computation, ACM, Beijing, China. URL: http://www.issac-conference.org/2019/software.php, https://math.ucas.ac.cn/index.php/zh-CN/xsyj/ 2427-a-package-to-compute-implicit-equations-for-rational-curves-and-surfaces.

Zapata, J.L.G., Martín, J.C.D., 2014. Finding the number of roots of a polynomial in a plane region using the winding number. Computers \& Mathematics with Applications 67, 555 - 568. URL: http://www.sciencedirect.com/science/article/pii/ S089812211300669X, doi:https://doi.org/10.1016/j.camwa.2013.11.013.

Zhao, X.Y., Zhu, C.G., 2015. Injectivity conditions of rational bzier surfaces. Computers \& Graphics 51, $17-25$. URL: http:// www.sciencedirect.com/science/article/pii/S0097849315000643, doi:https://doi.org/10.1016/j.cag.2015.05.017. international Conference Shape Modeling International.

Zhao, X.Y., Zhu, C.G., Wang, H., 2017. Geometric conditions of non-self-intersecting nurbs surfaces. Applied Mathematics and Computation 310, 89 - 96. URL: http://www.sciencedirect.com/science/article/pii/S0096300317302606, doi:https: //doi.org/10.1016/j.amc.2017.04.016. 